\title{
Toxoplasma's ROP-a-dope strategy
}

Intracellular pathogens insert many different proteins into their host cells, but the function and mechanism of action of these proteins are often poorly understood. In a recent issue of Structure, Labesse and colleagues describe the crystal structure of the secreted Toxoplasma gondii effector ROP2 and the implications for the parasite's interactions with the host cell.

Proteins of the ROP2 family (ROP2s) are secreted by T. gondii into the host cells, where they can subvert host cell responses. ROP2s are putative protein kinases, although kinase activity has not yet

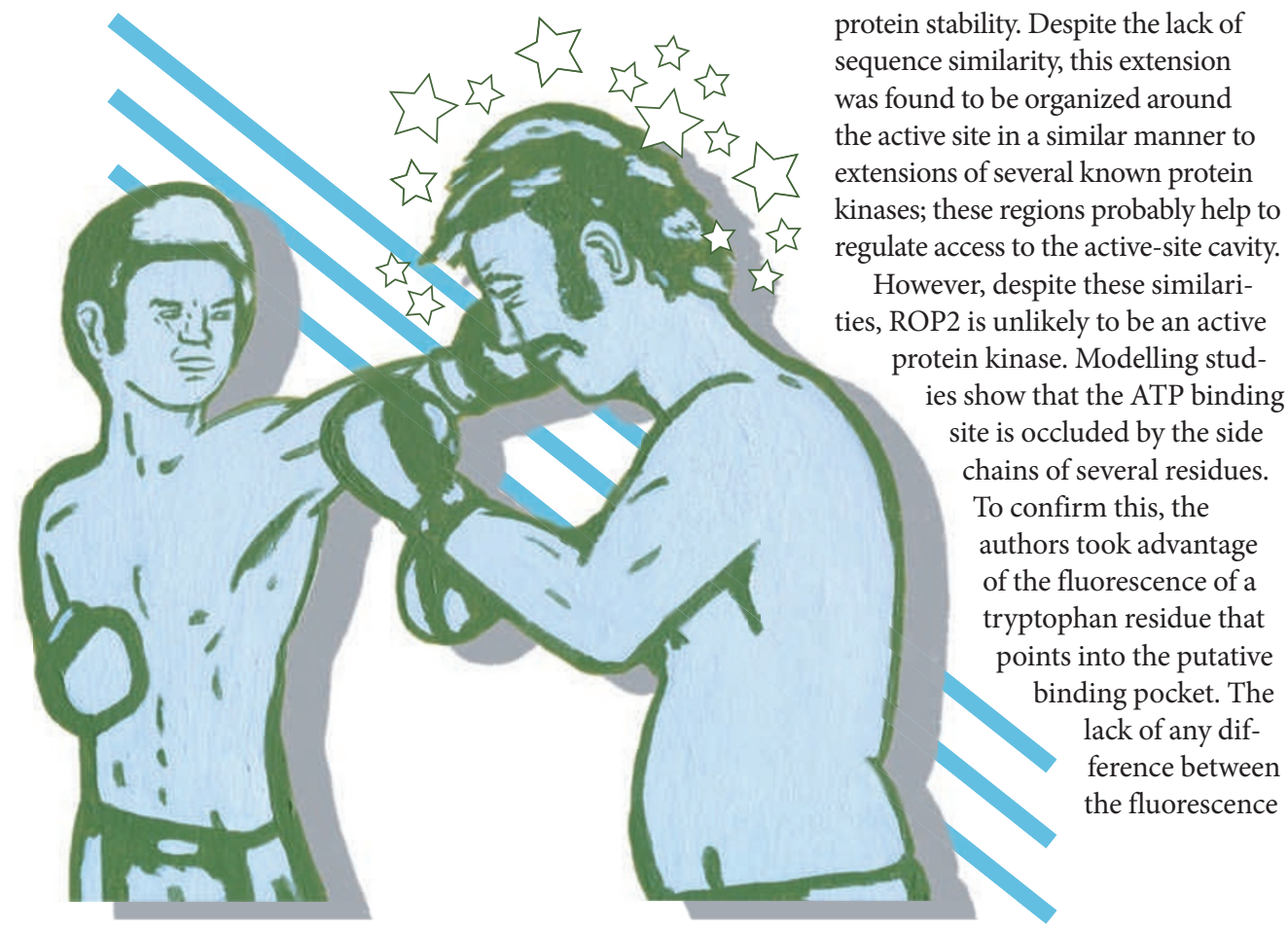

been established for every member. Labesse and colleagues determined the structure of ROP2 and found that the carboxy-terminal domain indeed bears similarity to protein kinases. However, sequence and structural conservation is limited. No hydrophobic or conserved patches were identified, preventing any prediction of protein-protein interactions or additional functions.

ROP2 family members contain conserved insertions, ranging from 8 to 18 amino acids, and a long amino-terminal extension. These interact with each other and probably provide additional stability; deletion of the $\mathrm{N}$-terminal extension decreased protein stability. Despite the lack of sequence similarity, this extension was found to be organized around the active site in a similar manner to extensions of several known protein kinases; these regions probably help to gulate access to the active-site cavity. However, despite these similariROP2 is unlikely to be an active otein kinase. Modelling studshow that the ATP binding ite is occluded by the side chains of several residues. To confirm this, the authors took advantage of the fluorescence of a tryptophan residue that ints into the putative ding pocket. The lack of any difference between the fluorescence levels of this residue in proteins incubated with or without ATP indicated that ATP does not enter the ATP-binding pocket. Similarly, NMR studies using ${ }^{15} \mathrm{~N}$-labelled ROP2 confirmed the absence of ATP binding.

Unlike the ATP-binding site, residues involved in peptide binding and the structure around these residues are well conserved in ROP2. The authors speculate that this region may retain protein-binding capabilities and that this is how ROP2 performs its function.

Several ROP2s bind to the parasitophorous vacuole membrane (PVM). Contrary to previous models, the authors suggest that membrane binding is mediated by short, amphipathic, but highly basic, regions upstream of the protein kinase fold. Experiments that used truncations of the ROP2 family member ROP18 showed that PVM association depended on an arginine-rich region and that this region was sufficient for PVM targeting. As the PVM is rich in negatively charged residues, this would provide specificity to the membrane binding.

The structure of ROP2 paves the way for further studies that could investigate the function and possible interacting partners of ROP2 and other ROP2s.

Christiaan van Ooij

ORIGINAL RESEARCH PAPER Labesse, G. et al. ROP2 from Toxoplasma gondii: a virulence factor with a protein-kinase fold and no enzymatic activity. Structure 17, 139-146 (2009) 